OPEN ACCESS

Edited by:

Maik Gollasch,

Charité Universitätsmedizin

Berlin, Germany

Reviewed by:

Gautam Bhave,

Vanderbilt University, United States

Tetsuhiro Tanaka,

The University of Tokyo, Japan

Swapnil Hiremath,

University of Ottawa, Canada

*Correspondence:

Johannes Stegbauer

johannes.stegbauer@med.

uni-duesseldorf.de

Specialty section: This article was submitted to

Nephrology,

a section of the journal

Frontiers in Medicine

Received: 25 November 2017 Accepted: 13 February 2018

Published: 09 March 2018

Citation:

Vonend O, Martin O, Rump LC, Kroepil $P$ and Stegbauer J (2018)

Erythrocyte Salt Sedimentation Assay Does Not Predict Response

to Renal Denervation.

Front. Med. 5:51.

doi: 10.3389/fmed.2018.00051

\section{Erythrocyte Salt Sedimentation Assay Does Not Predict Response to Renal Denervation}

\author{
Oliver Vonend ${ }^{1,2}$, Ole Martin ${ }^{2}$, Lars C. Rump ${ }^{2}$, Patrick Kroepil ${ }^{3}$ and Johannes Stegbauer ${ }^{2 *}$ \\ ${ }^{1}$ Nierenzentrum, DKD Helios Klinik Wiesbaden, Wiesbaden, Germany, ${ }^{2}$ Department of Nephrology, Medical Faculty, \\ University Hospital Düsseldorf, Heinrich-Heine-University Düsseldorf, Düsseldorf, Germany, ${ }^{3}$ Departement of Diagnostic and \\ Interventional Radiology, Medical Faculty, University Hospital Düsseldorf, Heinrich-Heine-University Düsseldorf, Düsseldorf, \\ Germany
}

Renal denervation (RDN) has recently been shown to be effective in patients without antihypertensive medication. However, about $30 \%$ of patients do not respond to RDN, and therefore, there exists a need to find predictors of response. Individuals are either salt-sensitive (SS) or non-salt-sensitive (NSS) in terms of their blood pressure (BP) regulation. The sympathetic nervous system can influence water and salt handling. RDN reduces sympathetic drive and has an impact on salt excretion. The present study was conducted to test the influence of salt sensitivity in terms of the BP reducing effect after RDN procedure. Salt sensitivity was estimated using the in vitro Erythrocyte Salt Sedimentation Assay (ESS). In 88 patients with resistant hypertension, RDN was performed. Office BP and lab testing were performed at baseline and at month 1, $3,6,12,18$, and 24 after RDN. A responder rate of $64.7 \%$ has been observed. Salt sensitivity measurements (ESS-Test) were completed in a subgroup of 37 patients with resistant hypertension. In this group, 15 were SS and 17 were salt-resistant according to the in vitro assay, respectively. The responder rate was 60\% in SS patients and $59.1 \%$ in NSS patients, respectively. Electrolytes as well as aldosterone and renin levels did not differ between the two groups at baseline and in the follow-up measurements. The present study showed that salt sensitivity, estimated using the ESS in vitro test, did not affect the outcome of RDN and, therefore, does not help to identify patients suitable for RDN.

Keywords: hypertension, renal denevation, salt sensitivity of blood pressure, salt sensitivity, blood pressure

\section{INTRODUCTION}

The important role of sodium in the development and maintenance of hypertension has been shown in numerous animal models and human studies (1). The sympathetic nervous system plays a pivotal role in sodium handling since sympathetic nerves can contribute to the physiology of salt-sensitive (SS) hypertension $(2,3)$. After an impressive start, renal denervation (RDN) struggled to show its effectiveness in the HTN-3 trial (4). The HTN-3 trial was the first blinded, randomized, and in particularly sham-controlled trial analyzing the effectiveness and safety of RDN. The office BP was lowered substantially by $-14 \pm 24 \mathrm{mmHg}$ after the RDN procedure. However, RDN failed to show its superiority to sham intervention since the drop of office $\mathrm{BP}$ was similarly in the control group without $\mathrm{RDN}$ (sham group $-12 \pm 26 \mathrm{mmhg}$ ). There were no major safety concerns reported in both groups. 
Several explanations were given to interpret the inhomogeneous results (5). Post hoc evaluation suggested adherence problems, insufficient $\mathrm{RDN}$, inclusion of patients with isolated systolic hypertension, and other factors. Some of these aspects have been addressed in the latest SPYRAL HTN-OFF MED trial design (6). Even before the HTN-3 trial was carried out, it was assumed that the office blood pressure (BP) reduction of the HTN-1 and -2 trials were overestimated due to a lack of double-blinding (7).

In the recent SPYRAL HTN-OFF MED study it has been shown, that RDN effectively reduces blood pressure in the absence of antihypertensive drugs in patients with an office systolic BP between 150 and $180 \mathrm{mmHg}$ in the absence of antihypertensive drugs (6). However, approximately one-third of patients did not respond to RDN in that trial.

Despite the above mentioned factors, not many valid parameters have been identified till now, which allow to predtict response to $\mathrm{RDN}$. In addition, the mechanisms how $\mathrm{RDN}$ reduces BP in man are not fully understood. Measuring an increased sympathetic activity at baseline (e.g. by norepinephrine spillover or muscle sympathetic nerve activity) would certainly be the gold standard to select patients with sympathetic overactivity for RDN (8). However, the methods described can hardly be integrated in the clinical routine. Therefore, it seems essential to find other easy-to-use predictors for a response to RDN. Evaluation of the salt sensitivity using the salt blood test (SBT) would be such an assay.

Recently, it has been shown in patients with resistant hypertension, that RDN might influence sodium excretion rate (9). In addition, patients with resistant hypertension are often characterized by an increased salt sensitivity (10).

In order to identify parameters that might predict $\mathrm{BP}$ response to RDN therapy, the influence of salt sensitivity were analyzed using an in vitro assay in a subgroup of 37 patients with resistant hypertension and RDN. This now commercially available assay could easily be integrated in the clinical workup before planning RDN. As described by the developer of the SBT, the erythrocyte sodium sensitivity (ESS), and the red blood cells (RBC) sodium buffer capacity does correspond to the patients' individual salt sensitivity $(11,12)$.

\section{MATERIALS AND METHODS}

In the years $2010-2015, n=88$ patients underwent RDN in a single center and were included for the present investigation. The patients received RDN in a specialized center (University Duesseldorf) to treat resistant hypertension. The patients were part of a local register (Ethic No. 3848) and/or included in the "GREAT Register" (13). The institutional review board of the Medical Faculty University of Düsseldorf approved this prospective study (NR 3848). A written informed consent was obtained from all participants of this study.

Only patients with long-lasting history of resistant hypertension were included. According to the current definition of resistant hypertension, patients with an office BP $>140 / 90 \mathrm{mmHg}$ with at least three antihypertensive drugs at maximal tolerated doses, of which one is diuretic were further evaluated for RDN. Before inclusion into the study, medical history including DM, cardiovascular risk factors, and OSAS were evaluated and secondary hypertension forms were excluded and medication was optimized according to the guidelines (14). RDN was performed as described elsewhere using the Medtronic Symplicity single electrode catheter $(4,15)$. The patients were evaluated at baseline prior RDN and 1, 3, 6, 12, 18, and 24 months after the procedure. Office BP (automated measurement, mean of three readings), ABPM and lab testing were performed at baseline and during follow-up. The blood test included hemoglobin, electrolytes, serum-creatinine (eGFR CKD-EPI), BUN, cystatin C, metanephrines, aldosterone, and renin. Urine sodium, potassium, urinecreatinine, and albumin were also measured.

Data (office BP and Lab values) of all 88 patients were available at baseline and at the 6 months follow-up; whereas only half of the patients had ABPM measurements due to lack of compliance. The number of available patient data at follow-up month 12,18 , and 24 were 76,64 , and 64 , respectively.

The presence of low or high salt sensitivity was prospectively tested in a subgroup of $n=37$ patients (years 2014-2015) using a recently established SBT before RDN therapy (11). In brief, red blood cells were suspended in stabilized solutions with different sodium concentrations. These ready to use solutions (SBT-KIT) were kindly provided by $\mathrm{H}$. Oberleithner of the University Muenster, Germany. The ratio of RBC sedimentation rates in high over low sodium solutions gives an estimate of individual ESS. ESS ratios $<4$ were defined as low SS, $>4$ as high SS, respectively.

A two-sided significance level of $p<0.05$ was applied to all calculations. Data were analyzed by IBM-SPSS ${ }^{\circledR}$ Statistics 20. Graphics were designed by SigmaPlot ${ }^{\circledR} 11.0$.

\section{RESULTS}

From the years 2010-2015, data of 88 consecutive patients collected at one single center were included for analysis. Table 1 lists the baseline characteristics. Median age was $59.7 \pm 10.8$. The average office BP at inclusion was $168.5 / 91.1 \pm 18.2 / 16.1 \mathrm{mmHg}$, the 24 -h ABP was $151.7 / 86.8 \pm 15.2 / 11.1 \mathrm{mmHg}$. The patients received at baseline a median of $5.6 \pm 1.4$ antihypertensive drugs. In a subgroup of $n=37$ patients, a SBT before RDN therapy was performed. Using this in vitro assay the patients were divided in SS and salt-resistant (SR).

The overall BP reduction after RDN is shown in Figures 1A,B. There was a significantly reduction of BP observed at all follow-up time points according to the office (Figure 1A) and $24 \mathrm{~h}$ BP (Figure 1B) readings. Six months after RDN the Office $\mathrm{BP}$ and the $24 \mathrm{~h}$ mean BP dropped by $-16.5 /-7.3 \mathrm{mmHg}$ and $-8.5 /-5.7 \mathrm{mmHg}$, respectively.

The overall responder rate after RDN was calculated to $64.7 \%$. A positive BP response to RDN was defined as follows: systolic office BP reduction of at least $10 \mathrm{mmHg}$ after 6 months (15). There were no significant differences between the $n=57$ responders and the $n=31$ non-responders in terms of their baseline characteristics except the following: in the responder group, there were more patients $<65$ years ( $72 \%$ responder vs. $58 \%$ non-responder; $p<0.05)$ and their systolic office BP was higher $(171.7 \pm 18.1$ vs. $162.5 \pm 17.2 ; p<0.05)$. 
TABLE 1 | Main baseline characteristics of treated patients and subgroup differentiation salt-sensitive (SS) vs. salt-resistant (SR) using the in vitro salt blood test.

\begin{tabular}{|c|c|c|c|c|}
\hline & All included pat. $(n=88)$ & Subgroup SS $(n=15)$ & Subgroup SR $(n=22)$ & SS vs. SR \\
\hline Age (years) & $59.7 \pm 10.8$ & $57.3 \pm 11$ & $63.7 \pm 9.2$ & $p=0.06$ \\
\hline$<65$ years & 58 (65.9\%) & $12(80.0 \%)$ & $11(50.0 \%)$ & $p=0.1$ \\
\hline Sex $m / f$ & $56 / 32$ & $9 / 6$ & $14 / 8$ & $p=0.9$ \\
\hline BMI $\left(\mathrm{kg} / \mathrm{m}^{2}\right)$ & $30.8 \pm 5.3$ & $31.4 \pm 5.5$ & $31.4 \pm 4.6$ & $p=0.9$ \\
\hline Antihypertensives & $5.6 \pm 1.4$ & $5.7 \pm 1.3$ & $5.5 \pm 1.9$ & $p=0.8$ \\
\hline Diabetes (all type) & $33(37.5 \%)$ & 7 (46.7\%) & $8(36.4 \%)$ & $p=0.6$ \\
\hline Obstructive sleep apnea & $56(63.6 \%)$ & $9(60.0 \%)$ & 19 (86.4\%) & $p=0.2$ \\
\hline Responder & $57(64.8 \%)$ & 9 (60.0\%) & $13(59.1 \%)$ & $p=0.9$ \\
\hline Office SBP & $168.5 \pm 18.2$ & $171.7 \pm 15.4$ & $167.5 \pm 16.6$ & $p=0.4$ \\
\hline Office DBP & $91.1 \pm 16.1$ & $91.8 \pm 14.8$ & $90.5 \pm 15.4$ & $p=0.8$ \\
\hline 24-h SBP & $151.7 \pm 15.2$ & $156.1 \pm 19.8$ & $157 \pm 14.9$ & $p=0.9$ \\
\hline 24-h DBP & $86.8 \pm 11.1$ & $88.9 \pm 8.3$ & $85.4 \pm 12.8$ & $p=0.4$ \\
\hline Creatinine (mg/dl) & $1.1 \pm 1.3$ & $1.0 \pm 0.4$ & $1.0 \pm 0.3$ & $p=0.8$ \\
\hline GFR CKD-EPI (ml/min/1.73 m²) & $79.2 \pm 23.5$ & $79.7 \pm 25.9$ & $74.5 \pm 19.2$ & $p=0.5$ \\
\hline Cystatin C (mg/l) & $1.0 \pm 0.6$ & $1.1 \pm 0.5$ & $1.0 \pm 0.3$ & $p=0.8$ \\
\hline eGFR (ml/min/1.73m²) & $93.3 \pm 36.2$ & $81.3 \pm 30.3$ & $86.6 \pm 24.4$ & $p=0.6$ \\
\hline Urea (mg/dl) & $40.4 \pm 19$ & $38.6 \pm 11.9$ & $38 \pm 15.6$ & $p=0.9$ \\
\hline Microalbuminuria (mg/dl) & $167.7 \pm 439.4$ & $201.3 \pm 347.2$ & $98.6 \pm 240.7$ & $p=0.3$ \\
\hline Sodium $(\mathrm{mmol} / \mathrm{l})$ & $141.9 \pm 3$ & $141.9 \pm 2.5$ & $141.3 \pm 2.6$ & $p=0.5$ \\
\hline Potassium (mmol/l) & $4.0 \pm 0.5$ & $4.0 \pm 0.4$ & $4.0 \pm 0.4$ & $p=0.7$ \\
\hline Hemoglobin (g/dl) & $14.0 \pm 1.3$ & $13.9 \pm 0.9$ & $14.2 \pm 1.3$ & $p=0.5$ \\
\hline Glucose (mg/dl) & $130.9 \pm 38.6$ & $133.6 \pm 41.9$ & $143 \pm 55$ & $p=0.6$ \\
\hline $\mathrm{HbA} 1 \mathrm{c}(\%)$ & $6.2 \pm 1.1$ & $6.5 \pm 1.6$ & $6.4 \pm 1.5$ & $p=0.8$ \\
\hline Insulin (mU/I) & $24.1 \pm 27.7$ & $32.0 \pm 52$ & $22.1 \pm 14.9$ & $p=0.4$ \\
\hline c-Peptide $(\mu \mathrm{g} / \mathrm{l})$ & $4.2 \pm 2.6$ & $4.6 \pm 3.6$ & $4.1 \pm 1.9$ & $p=0.6$ \\
\hline Aldosterone (pg/ml) & $102.7 \pm 66.7$ & $77 \pm 54.1$ & $114.1 \pm 74.8$ & $p=0.1$ \\
\hline $\operatorname{Renin}(\mathrm{pg} / \mathrm{ml})$ & $63 \pm 200.4$ & $22.8 \pm 32.2$ & $14.9 \pm 28.5$ & $p=0.5$ \\
\hline Metanephrine (ng/l) & $47.4 \pm 23.3$ & $38.8 \pm 14.2$ & $42.5 \pm 15.4$ & $p=0.5$ \\
\hline Normetanephrine (ng/l) & $79.2 \pm 33.7$ & $73.0 \pm 20.1$ & $64.8 \pm 25.3$ & $p=0.3$ \\
\hline Cholesterol (mg/dl) & $199 \pm 40.6$ & $195.5 \pm 27$ & $194.9 \pm 41.9$ & $p=0.9$ \\
\hline $\mathrm{HDL}(\mathrm{mg} / \mathrm{dl})$ & $49.8 \pm 15.1$ & $44.8 \pm 14.5$ & $49.0 \pm 13.5$ & $p=0.4$ \\
\hline LDL (mg/dl) & $128.8 \pm 38.5$ & $127.1 \pm 26.7$ & $128.1 \pm 51.8$ & $p=0.9$ \\
\hline NT-proBNP (pg/ml) & $307 \pm 504.6$ & $586.2 \pm 960.1$ & $266.5 \pm 447.6$ & $p=0.2$ \\
\hline
\end{tabular}

Data are $n$ (\%) or mean (SD). BMI, body-mass index; SBP, systolic blood pressure; DBP, diastolic blood pressure; GFR, glomerular filtration rate; eGFR, estimated GFR; HbA1C, glycosylated hemoglobin; HDL, high density lipoprotein; LDL, low density lipoprotein; NT-proBNP, N-terminal pro-brain natriuretic peptide.

A subgroup analysis separating patients below and above 65 years could demonstrate that younger patients respond better to RDN (Figure 2). Six months after RDN, the diastolic office BP dropped significantly in the patients younger than 65 years but not in the group of patients $>65$ years of age $(-10.2 \pm 4.1 \mathrm{mmHg}$ vs. $-1.6 \pm 3.6 \mathrm{mmHg}$ in $<65$ vs. $>65$ years).

For impending analyses, the patients were allocated in SS and non-salt-sensitive (NSS) according to their salt sensitivity testing results. As shown in Table 1, no significant differences including age were detected between both groups. Subgroup allocation was done according to the SBT groups. ESS ratios $<4$ were defined as low SS, $>4$ as high SS. Accordingly, the ESS ratios were significantly different between both groups. The mean ESS ratio in the SS group was $6.5 \pm 1.6$ vs. $3.0 \pm 0.7$ in the SR group $(p<0.01)$

\section{Response to RDN According Salt Sensitivity}

The BP reducing effect of RDN did not differ significantly between both groups. The responder rates (SBP reduction $\geq 10 \mathrm{mmHg}$ 6 months after RDN) were 60.0 and $59.1 \%$ in SS and SR patients, respectively. The progression in office $\mathrm{BP}$ after $\mathrm{RDN}$ within the follow-up of 24 months was similar in both groups (Figure 3). There was no statistical difference in the BP lowering effect responding to RND between SS and SR patients at month 6 $(28 \pm 16$ vs. $23 \pm 9 \mathrm{mmHg})$. Successful ABPM readings at month 0 and 6 were available for $n=24$ patients. There were no differences in baseline (Table 1) and follow-up mean values apparent (24 h mean SS: month 6: 147/83 \pm 6/4 mmHg; SR: month 6: $144 / 77 \pm 6 / 3 \mathrm{mmHg}$ ).

There is no apparent correlation between the salt sensitivity according ESS-Testing and the BP response to RDN as shown by systolic office and $24 \mathrm{~h} \mathrm{BP}$ measurements at the 6 months followup (Figure 4).

In Table 2 the data on urinary sodium excretion in both SR/ SS and responder/non-responder groups are shown. There are no difference between both groups and no difference within the groups, respectively.

\section{DISCUSSION}

Renal denervation just recently came back into focus, since the latest randomized, sham-controlled trial was able to show a benefit of the procedure (6). Numerous preclinical studies showed, that sympathetic nerve activity plays a major role in the development of hypertension. In human clinical trials there are numerous factors, like inhomogeneous patient characteristics, 


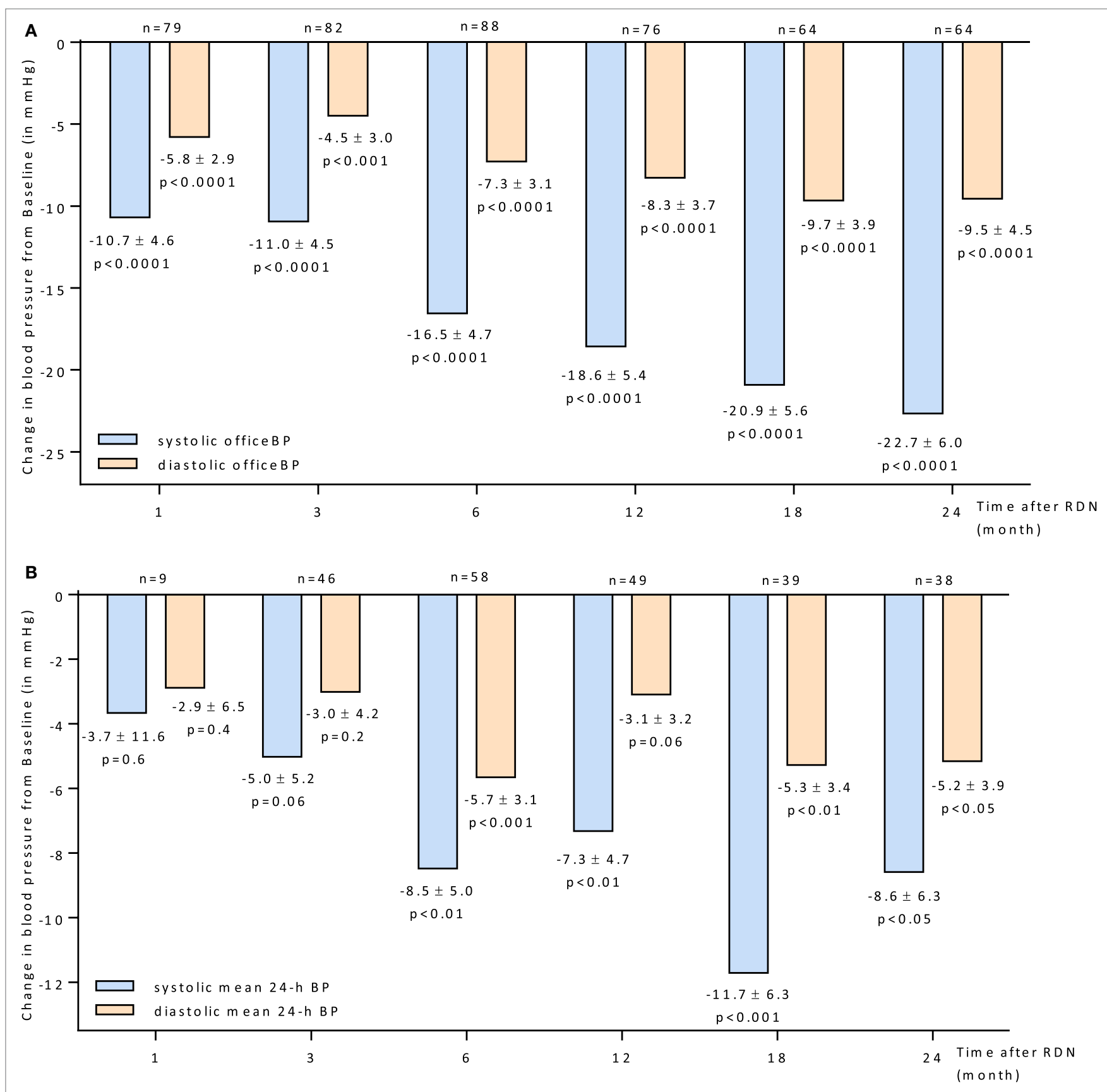

FIGURE 1 | (A) Changes up to 24 months in systolic and diastolic office BP for all patients $95 \%$ Cls and unadjusted $p$ values shown. (B) Changes up to 24 months in systolic and diastolic mean $24-\mathrm{h}$ BP for all patients $95 \% \mathrm{Cls}$ and unadjusted $p$ values shown. Abbreviations: RDN, renal denervation; BP, blood pressure.

adherence problems, procedural weakness and other confounders that can dilute and even eliminate the anticipated effect as it happened in the past (4).

In this single center analysis, a considerable proportion of patients benefited from RDN. As also shown in other trials particularly patients with the high baseline BP are more likely to respond (16). The responder rate of $64.7 \%$ in this analysis and the overall $\mathrm{BP}$ reduction of $-17 /-7 \mathrm{mmHg}$ in office $\mathrm{BP}$ and $-8 /-6 \mathrm{mmHg}$ in $24 \mathrm{~h}$ mean $\mathrm{BP}$ accordingly, was comparable to other RDN trials (16-19). In the recently published Austrian Transcatheter National Multicentre Renal Denervation (TREND) Registry, Zweiker and colleagues found very similar results in their $188 \mathrm{RDN}$ patients treated in 14 centers. A reduction of $-20 /-7 \mathrm{mmHg}$ in office BP and $-8 /-5 \mathrm{mmHg}$ in $24 \mathrm{~h}$ mean BP was described (16). The large Global Symplicity Registry that includes the German GREAT Registry showed in their set of 998 patients treated in 134 centers a reduction of $-12 \mathrm{mmHg}$ in office BP 6 months after RDN (ABPM reduction $-7 \mathrm{mmHg}$ ) (13). 


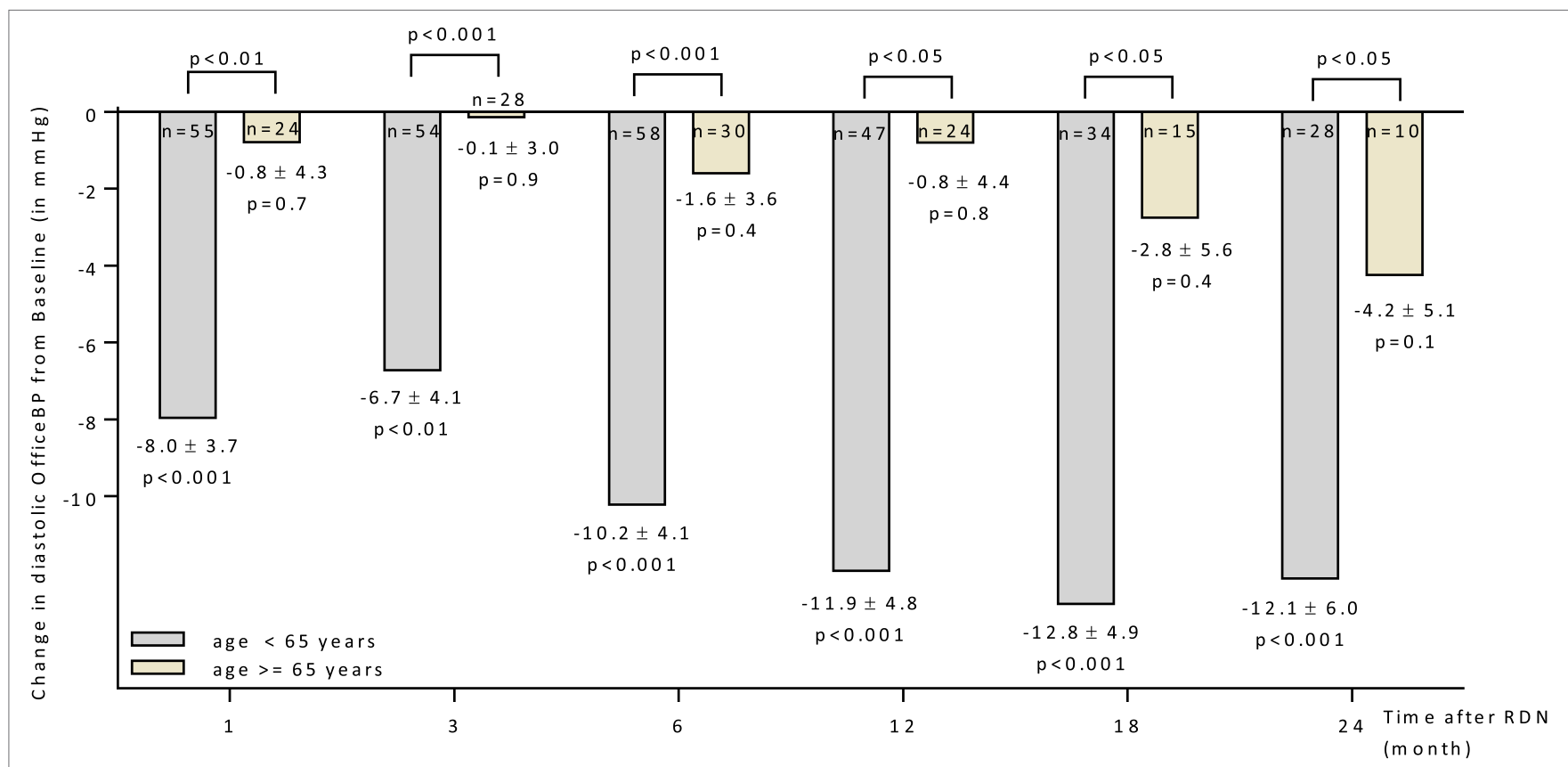

FIGURE 2 | Changes up to 24 months in diastolic office blood pressure for patients age above and below 65 years. 95\% Cls and unadjusted $p$ values shown. Abbreviations: RDN, renal denervation; OfficeBP, office blood pressure.

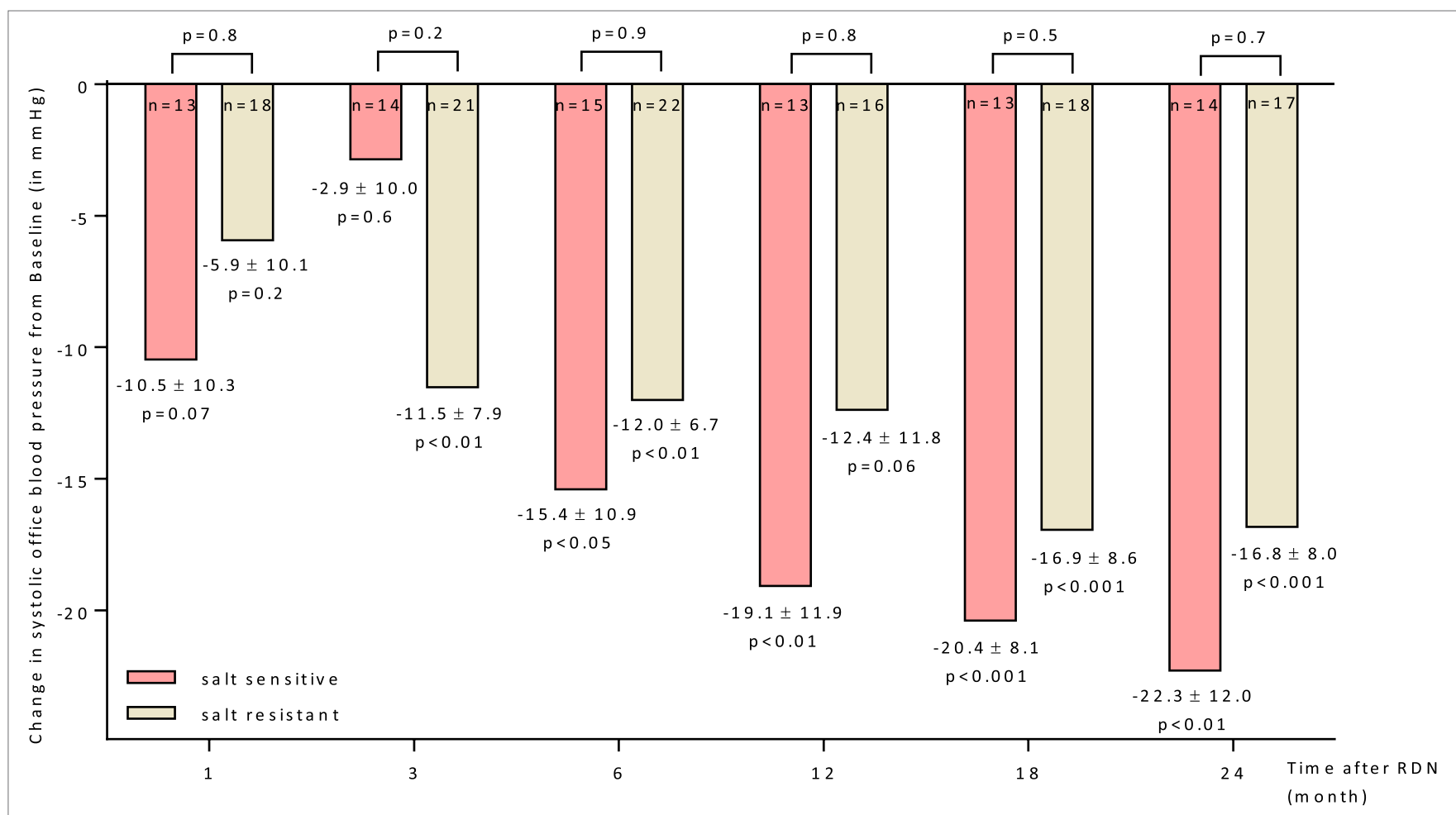

FIGURE 3 | Changes up to 24 months in systolic office blood pressure for salt-sensitive and salt-resistant groups. 95\% Cls and unadjusted $p$ values shown. Abbreviation: RDN, renal denervation.

As observed by others, older patients with high central aortic $\mathrm{BP}$ or isolated systolic hypertension tend to be less susceptible to $\operatorname{RDN}(20,21)$. Correspondingly, patients at an age $>65$ years did not respond well to RDN in our study. In older patients with a long standing arterial hypertension resulting in a higher arterial stiffness with an enlarged pulse-pressure (blood pressure 


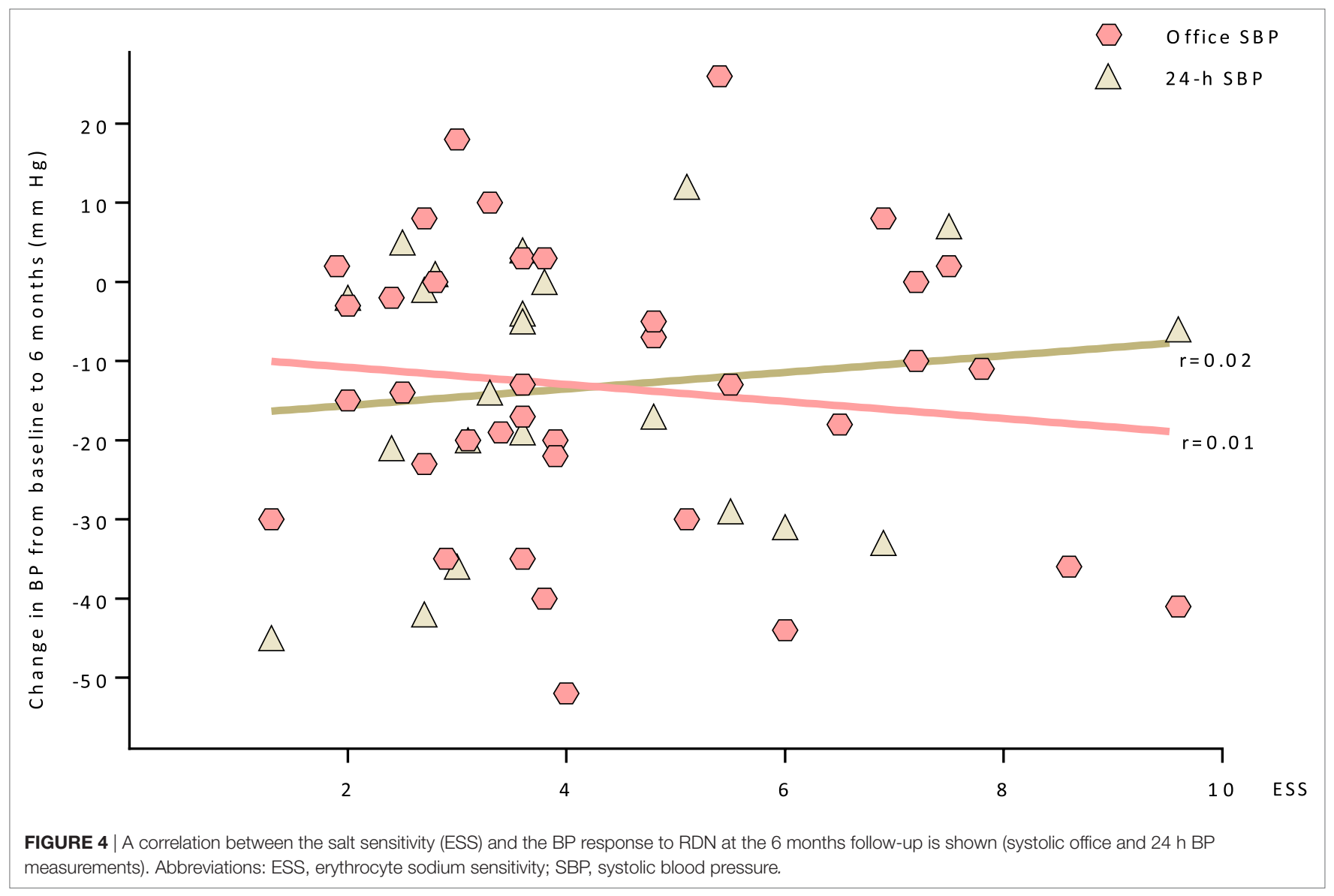

TABLE 2 | The urinary sodium excretion in salt-resistant (SR) and salt-sensitive (SS) patients as well as responder and non-responder groups are shown.

\begin{tabular}{|c|c|c|c|c|c|c|}
\hline & SR & ss & & Responder & Non-Responder & \\
\hline 1 Month $(n=27)$ & $101.5 \pm 45.9$ & $80.0 \pm 29.5$ & $p=0.2$ & $96.3 \pm 41.1$ & $98.5 \pm 40.9$ & $p=0.9$ \\
\hline 6 Month $(n=20)$ & $94.7 \pm 45.8$ & $131.7 \pm 46.5$ & $p=0.1$ & $103.6 \pm 42.4$ & $103.6 \pm 42.9$ & $p=1.0$ \\
\hline 12 Month $(n=26)$ & $97.1 \pm 33.5$ & $108.1 \pm 39.9$ & $p=0.5$ & $101.1 \pm 41.1$ & $101.6 \pm 37.3$ & $p=1.0$ \\
\hline 18 Month $(n=14)$ & $91.7 \pm 20.6$ & $130.0 \pm 73.8$ & $p=0.4$ & $91.6 \pm 29.9$ & $127.2 \pm 43.2$ & $p=0.7$ \\
\hline 24 Month $(n=9)$ & $107.3 \pm 31.1$ & $121.3 \pm 58.7$ & $p=0.7$ & $115.4 \pm 44.6$ & $103.3 \pm 34.9$ & $p=0.8$ \\
\hline
\end{tabular}

No statistical differences between the groups were apparent (Wilcoxon- and Mann-Whitney Test).

Unit: $m$ mol/l; values in mean $\pm S D$.

amplitude) the role of the sympathetic nervous system in driving the elevated BP seems to be less important.

To find other factors predicting response besides age and BP, salt sensitivity was tested prior $\mathrm{RDN}$ for the first time. It was hypothesized, that being SS might have an impact on the response to RDN. The particular effect of renal nerves on the regulation of arterial BP and sodium balance is still not fully understood (22). Various indicators of sympathetic drive can be altered by RDN. Dorr and colleagues found a correlation of BP response to RDN and reduction in the level of the sympathetic cotransmitter Neuropeptide Y (23). Recently, Poss and coworkers were able to demonstrate a positive effect of RDN on sodium excretion (9). Noradrenaline, the most important sympathetic neurotransmitter modulates salt handling and, therefore, regulates BP though different mechanisms.

Thus, it has been shown that stimulation of alpha and beta adrenergic receptors directly activates the thiazide-sensitive $\mathrm{NaCl}$ cotransporter leading to sodium reabsorption (1). In addition, sympathetic neurotransmitters increase renal vascular resistance and induce renin release thereby regulating water and salt balance $(22,24)$. Despite this overwhelming evidence for an important role of renal sympathetic nerve activity on salt handling, the present study demonstrated that salt sensitivity does not seem to influence the BP response to RDN. Moreover, the blood pressure reduction in patients responding to RDN was similar between SS and NSS indicating that salt sensitivity did not affect the outcome 
after RDN. Therefore, salt sensitivity seems not to be a suitable predictor for successful RDN.

As mentioned in the Section "Introduction," there seems to be a relation between the salt sensitivity in man and arterial hypertension. However it has to be mentioned, that this pathophysiologic relationship has been as yet not fully investigated in clinical trials with large patient numbers. Therefore, it is unknown whether salt sensitivity measured by the SBT could be an useful predictor for selecting hypertensive patients who will respond to RDN. This should be investigated in further studies. Larger trials confirming the clear correlation between the SBT and the renal sodium handling and excretion or response to sodium loading are missing and need to follow.

The HTN-3 trial subgroup analysis might support this observation. The response to $\mathrm{RDN}$ in the rather SS AfroAmerican population was not more pronounced compared to Caucasians (5).

Renal denervation fails to reduce BP in $20-40 \%$ of patients. Auxiliary predictors for response could not be identified yet. The present study suggests that salt sensitivity, measured using the in vitro assay SBT, does not influence the response to RDN.

\section{REFERENCES}

1. Blaustein MP, Leenen FH, Chen L, Golovina VA, Hamlyn JM, Pallone TL, et al. How $\mathrm{NaCl}$ raises blood pressure: a new paradigm for the pathogenesis of salt-dependent hypertension. Am J Physiol Heart Circ Physiol (2012) 302(5):H1031-49. doi:10.1152/ajpheart.00899.2011

2. Foss JD, Fink GD, Osborn JW. Reversal of genetic salt-sensitive hypertension by targeted sympathetic ablation. Hypertension (2013) 61(4):806-11. doi:10.1161/HYPERTENSIONAHA.111.00474

3. Fujita M, Fujita T. The role of CNS in salt-sensitive hypertension. Curr Hypertens Rep (2013) 15(4):390-4. doi:10.1007/s11906-013-0358-z

4. Bhatt DL, Kandzari DE, O’Neill WW, D’Agostino R, Flack JM, Katzen BT, et al. A controlled trial of renal denervation for resistant hypertension. N Engl J Med (2014) 370(15):1393-401. doi:10.1056/NEJMoa1402670

5. Kandzari DE, Bhatt DL, Brar S, Devireddy CM, Esler M, Fahy M, et al. Predictors of blood pressure response in the SYMPLICITY HTN-3 trial. Eur Heart J (2014) 36(4):219-27. doi:10.1093/eurheartj/ehu441

6. Townsend RR, Mahfoud F, Kandzari DE, Kario K, Pocock S, Weber MA, et al. Catheter-based renal denervation in patients with uncontrolled hypertension in the absence of antihypertensive medications (SPYRAL HTN-OFF MED): a randomised, sham-controlled, proof-of-concept trial. Lancet (2017) 390(10108):2160-70. doi:10.1016/S0140-6736(17)32281-X

7. Howard JP, Nowbar AN, Francis DP. Size of blood pressure reduction from renal denervation: insights from meta-analysis of antihypertensive drug trials of 4,121 patients with focus on trial design: the CONVERGE report. Heart (2013) 99(21):1579-87. doi:10.1136/heartjnl-2013-304238

8. Schlaich MP, Sobotka PA, Krum H, Lambert E, Esler MD. Renal sympathetic-nerve ablation for uncontrolled hypertension. N Engl J Med (2009) 361(9):932-4. doi:10.1056/NEJMc0904179

9. Poss J, Ewen S, Schmieder RE, Muhler S, Vonend O, Ott C, et al. Effects of renal sympathetic denervation on urinary sodium excretion in patients with resistant hypertension. Clin Res Cardiol (2015) 104(8):672-8. doi:10.1007/ s00392-015-0832-5

10. Calhoun DA, Jones D, Textor S, Goff DC, Murphy TP, Toto RD, et al. Resistant hypertension: diagnosis, evaluation, and treatment. A scientific statement from the American Heart Association Professional Education Committee of the Council for High Blood Pressure Research. Hypertension (2008) 51(6):1403-19. doi:10.1161/HYPERTENSIONAHA.108.189141

11. Oberleithner H, Wilhelmi M. Determination of erythrocyte sodium sensitivity in man. Pflugers Arch (2013) 465(10):1459-66. doi:10.1007/s00424-013-1289-x
The small sample size of the presented data is certainly a limitation of this analysis. In addition, the in vitro assay SBT can only estimate the salt sensitivity of the tested patients. A larger trial with conventional salt loading would be needed for more dependable results. We have to continue to study the underlying mechanism how RDN interacts with $\mathrm{BP}$ control in order to identify suitable patients for this therapy. Differentiation between SS and not SS does not seem to be helpful in predicting response to RDN.

\section{ETHICS STATEMENT}

Ethic commitee University Duesseldorf Ethic No. 3848.

\section{AUTHOR CONTRIBUTIONS}

OV conducted the trial and has written the manuscript. OM collected the trial data, performed statistical analyses, and arranged the graphics. LR has written parts of the manuscrips, edited the manuscript. PS collected trial data and performed renal denervation. JS conducted the trial and has written the manuscript.

12. Oberleithner H, Wilhelmi M. Salt sensitivity determined from capillary blood. Kidney Blood Press Res (2016) 41(4):355-64. doi:10.1159/0004 43438

13. Bohm M, Mahfoud F, Ukena C, Hoppe UC, Narkiewicz K, Negoita M, et al. First report of the Global SYMPLICITY Registry on the effect of renal artery denervation in patients with uncontrolled hypertension. Hypertension (2015) 65(4):766-74. doi:10.1161/HYPERTENSIONAHA.114.05010

14. Mancia G, Fagard R, Narkiewicz K, Redon J, Zanchetti A, Bohm M, et al. 2013 ESH/ESC Guidelines for the management of arterial hypertension: the Task Force for the management of arterial hypertension of the European Society of Hypertension (ESH) and of the European Society of Cardiology (ESC). J Hypertens (2013) 31(7):1281-357. doi:10.1097/01.hjh.0000431740. 32696.cc

15. Esler MD, Krum H, Sobotka PA, Schlaich MP, Schmieder RE, Bohm M. Renal sympathetic denervation in patients with treatment-resistant hypertension (The Symplicity HTN-2 Trial): a randomised controlled trial. Lancet (2010) 376(9756):1903-9. doi:10.1016/S0140-6736(10)62039-9

16. Zweiker D, Lambert T, Steinwender C, Weber T, Suppan M, Brussee H, et al. Effects of renal denervation documented in the Austrian National Multicentre Renal Denervation Registry. PLoS One (2016) 11(8):e0161250. doi:10.1371/ journal.pone.0161250

17. Lambert T, Blessberger H, Gammer V, Nahler A, Grund M, Kerschner K, et al. Effects of renal denervation on ambulatory blood pressure measurements in patients with resistant arterial hypertension. Clin Cardiol (2014) 37(5):307-11. doi:10.1002/clc. 22269

18. Mahfoud F, Ukena C, Schmieder RE, Cremers B, Rump LC, Vonend O, et al. Ambulatory blood pressure changes after renal sympathetic denervation in patients with resistant hypertension. Circulation (2013) 128(2):132-40. doi:10.1161/CIRCULATIONAHA.112.000949

19. Persu A, Jin Y, Azizi M, Baelen M, Volz S, Elvan A, et al. Blood pressure changes after renal denervation at 10 European expert centers. J Hum Hypertens (2014) 28(3):150-6. doi:10.1038/jhh.2013.88

20. Ewen S, Ukena C, Linz D, Kindermann I, Cremers B, Laufs U, et al. Reduced effect of percutaneous renal denervation on blood pressure in patients with isolated systolic hypertension. Hypertension (2015) 65(1):193-9. doi:10.1161/ HYPERTENSIONAHA.114.04336

21. Ott C, Schmid A, Toennes SW, Ditting T, Veelken R, Uder M, et al. Central pulse pressure predicts BP reduction after renal denervation in patients with treatment-resistant hypertension. EuroIntervention (2015) 11(1):110-6. doi:10.4244/EIJV11I1A19 
22. DiBona GF, Esler M. Translational medicine: the antihypertensive effect of renal denervation. Am J Physiol Regul Integr Comp Physiol (2010) 298(2):R245-53. doi:10.1152/ajpregu.00647.2009

23. Dorr O, Ewen S, Liebetrau C, Mollmann H, Gaede L, Linz D, et al. Neuropeptide $\mathrm{Y}$ as an indicator of successful alterations in sympathetic nervous activity after renal sympathetic denervation. Clin Res Cardiol (2015) 104(12):1064-71. doi:10.1007/s00392-015-0874-8

24. Sparks MA, Stegbauer J, Chen D, Gomez JA, Griffiths RC, Azad HA, et al. Vascular type 1A angiotensin II receptors control BP by regulating renal blood flow and urinary sodium excretion. J Am Soc Nephrol (2015) 26(12):2953-62. doi:10.1681/ASN.2014080816
Conflict of Interest Statement: The authors declare that the research was conducted in the absence of any commercial or financial relationships that could be construed as a potential conflict of interest.

Copyright (C) 2018 Vonend, Martin, Rump, Kroepil and Stegbauer. This is an open-access article distributed under the terms of the Creative Commons Attribution License (CC BY). The use, distribution or reproduction in other forums is permitted, provided the original author(s) and the copyright owner are credited and that the original publication in this journal is cited, in accordance with accepted academic practice. No use, distribution or reproduction is permitted which does not comply with these terms. 